\title{
Gut Bacterial Dysbiosis and Instability is Associated with the Onset of
}

\section{Complications and Mortality in COVID-19}

\section{David Schult $^{1 *}$, Sandra Reitmeier ${ }^{2,3 *}$, Plamena Koyumdzhieva ${ }^{1 *}$, Tobias Lahmer ${ }^{1}$, Moritz} Middelhoff $^{1}$, Johanna Erber ${ }^{1}$, Jochen Schneider ${ }^{1}$, Juliane Kager ${ }^{1}$, Marina Frolova ${ }^{1}$, Julia Horstmann $^{1}$, Lisa Fricke ${ }^{1}$, Katja Steiger ${ }^{4}$, Moritz Jesinghaus ${ }^{4,5}$, Klaus-Peter Janssen ${ }^{6}$, Ulrike Protzer $^{7}$, Klaus Neuhaus ${ }^{2}$, Roland M. Schmid ${ }^{1}$, Dirk Haller ${ }^{2,3}$, Michael Quante ${ }^{1,8}$

\section{*shared co-first authorship.}

${ }^{1}$ Department of Internal Medicine II, Klinikum rechts der Isar, Technische Universität München, Munich, Germany; ${ }^{2}$ ZIEL - Institute for Food \& Health, Technische Universität München, Freising, Germany; ${ }^{3}$ Chair of Nutrition and Immunology, Technische Universität München, Freising, Germany; ${ }^{4}$ Institute of Pathology, Technische Universität München, Munich, Germany; ${ }^{5}$ Institute of Pathology, University Hospital Marburg, Marburg, Germany; ${ }^{6}$ Department of Surgery, Klinikum rechts der Isar, Technische Universität München, Munich, Germany; ${ }^{7}$ Institute of Virology, Technische Universität/Helmholtz Zentrum München, Munich, Germany; ${ }^{8}$ Department of Internal Medicine II, Universitätsklinikum Freiburg, Universität Freiburg, Freiburg, Germany.

Correspondence to: Michael Quante, Klinik für Innere Medizin II, Universität Freiburg, Hugstetter Str. 55, Freiburg 79106, Germany. Phone: 49.761.270.32766; Email: michael.quante@uniklinik-freiburg.de

\section{Word Count: 3977}




\section{Abstract}

Objective: There is a growing debate about the involvement of the gut microbiome in COVID19 , although it is not conclusively understood whether the microbiome has an impact on COVID-19, or vice versa, especially as analysis of amplicon data in hospitalized patients requires sophisticated cohort recruitment and integration of clinical parameters. Here, we analyzed fecal and saliva samples from SARS-CoV-2 infected and post COVID-19 patients and controls considering multiple influencing factors during hospitalization. Design: 16S rRNA gene sequencing was performed on fecal and saliva samples from 108 COVID-19 and 22 post COVID-19 patients, 20 pneumonia controls and 26 asymptomatic controls. Patients were recruited over the first and second corona wave in Germany and detailed clinical parameters were considered. Serial samples per individual allowed intra-individual analysis. Results: We found the gut and oral microbiota to be altered depending on number and type of COVID-19associated complications and disease severity. The occurrence of individual complications was correlated with low-risk (e.g., Faecalibacterium prausznitzii) and high-risk bacteria (e.g., Parabacteroides). We demonstrated that a stable gut bacterial composition was associated with a favorable disease progression. Based on gut microbial profiles, we identified a model to estimate mortality in COVID-19. Conclusion: Gut microbiota are associated with the occurrence of complications in COVID-19 and may thereby influencing disease severity. A stable gut microbial composition may contribute to a favorable disease progression and using bacterial signatures to estimate mortality could contribute to diagnostic approaches. Importantly, we highlight challenges in the analysis of microbial data in the context of hospitalization. 
The global pandemic caused by the new severe acute respiratory syndrome coronavirus 2 (SARS-CoV-2) brought the health systems to its limitations. The disease is characterized by respiratory symptoms although there is increasing evidence of gastrointestinal (GI) tract involvement $[1,2,3]$. At 15-39\%, nausea, vomiting, and diarrhea are relatively common in COVID-19[4] and a proportion of patients reports only gastrointestinal symptoms[5]. The virus itself is not limited to the lungs but replicates in human enterocytes[6] and is detectable in the patients' fecal samples[1, 7]. GI symptoms in patients with COVID-19 appear to be associated with increased disease severity and complications[8], although the underlying causes are not understood. Recent studies suggest that an altered microbial composition correlates with COVID-19 disease severity and inflammatory response to the disease[9, 10].

Common complications of COVID-19 include venous thromboembolism[11, 12], hemodynamic instability[13], and acute kidney injury[14]. Particularly in severe cases, an excessive and prolonged immune response to the virus is thought to be a catalyst of severity[15, 16].

The composition of the gut microbiota plays a critical role in the immunological homeostasis of the human body[17, 18]. It is known that the microbiome of the human gut is sensitive to changes in the hosts' environment[19]. In addition to antibiotic use, diet[20], and geographical differences[21, 22], critically ill patients show a rapid depletion of health-promoting organisms[23].

The study examined the impact of gut and oral microbiota on complication rate and outcome and, conversely, how hospitalization affects the gut microbial composition in this cohort. 
80

The study population consists of 4 groups: (1) 108 patients with laboratory confirmed SARSCoV-2 infection, (2) 22 patients post COVID-19 who had cleared the virus and were tested negative at first sampling, (3) 20 symptomatic pneumonia controls (SC) and (4) 26 age and gender matched asymptomatic controls (AC) (Table 1, Figure 1 A). Altogether, 251 stool samples and 160 saliva samples were examined. Serial samples were collected to investigate intra-individual changes over time. A total of 25 and 15 COVID-19 patients, 11 and 5 post COVID-19 patients and 3 and 2 SC provided serial stool and saliva samples, respectively (Table 1). The SC patients were admitted with respiratory symptoms of community-acquiredpneumonia (CAP) and were tested negative for SARS-CoV-2. Patients in the AC group were considered SARS-CoV-2 negative as they presented mainly for screening colonoscopy and showed no symptoms of SARS-CoV-2 infection. To minimize potential influencing factors on the microbiota in the AC cohort, patients with active cancer, inflammatory bowel disease (IBD), oncologic therapy or antibiotic intake at the time of examination or within 6 months prior were excluded. Endoscopic examination and pathology reports from colon biopsies had to be unremarkable.

\begin{tabular}{l|llll} 
& COVID-19 & POST COVID-19 & SC & AC \\
\hline NUMBER OF PATIENTS $(N)$ & 108 & 22 & 20 & 26 \\
STOOL SAMPLES $(N)$ & 150 & 60 & 15 & 26 \\
PATIENTS WITH SERIAL STOOL SAMPLES $(N)$ & 25 & 11 & 3 & 0 \\
SALIVA SAMPLES $(N)$ & 117 & 24 & 19 & 0 \\
PATIENTS WITH SERIAL SALIVA SAMPLES $(N)$ & 15 & 5 & 2 & 0 \\
GENDER $(F E M A L E S: M A L E S)$ & $49: 59$ & $4: 18$ & $5: 15$ & $11: 15$ \\
AGE $($ YEARS, MEAN, SD) & $62(15)$ & $65(13)$ & $64(17)$ & $63(12)$ \\
COMORBIDITIES $(N, \%)$ & & & \\
HYPERTENSION & $43(39.8)$ & $14(63.6)$ & $9(45)$ & $4(15.4)$ \\
DIABETES MELLITUS II & $19(17.6)$ & $5(22.7)$ & $3(15)$ & $3(11.5)$ \\
CORONARY HEART DISEASE & $16(14.8)$ & $3(13.6)$ & $8(40)$ & $1(3.8)$ \\
CHRONIC KIDNEY DISEASE & $9(8.3)$ & $7(31.8)$ & $3(15)$ & $1(3.8)$ \\
CANCER & $9(8.3)$ & $3(13.6)$ & $5(25)$ & $0(0)$ \\
CHRONIC OBSTRUCTIVE LUNG DISEASE & $5(4.6)$ & $1(4.5)$ & $1(5)$ & $0(0)$ \\
CHRONIC HEART FAILURE & $5(4.6)$ & $0(0)$ & $4(20)$ & $0(0)$ \\
DIVERTICULAR DISEASE & $4(3.7)$ & $1(4.5)$ & $0(0)$ & $12(46)$ \\
S.P. INTESTINAL RESECTION & $4(3.7)$ & $0(0)$ & $0(0)$ & $1(3.8)$ \\
RHEUMATIC DISEASE & $4(3.7)$ & $2(9)$ & $1(5)$ & $1(3.8)$ \\
INFLAMMATORY BOWEL DISEASE & $3(2.8)$ & $0(0)$ & $0(0)$ & $0(0)$ \\
GASTRITIS & $3(2.8)$ & $1(4.5)$ & $0(0)$ & $4(15.4)$ \\
REFLUX DISEASE & $2(1.9)$ & $1(4.5)$ & $0(0)$ & $1(3.8)$ \\
SYMPTOMS AT ADMISSION $(N, \%)$ & & & & $0(0)$ \\
COUGH & $69(63.9)$ & $13(5.9)$ & $7(35)$ & $0(0)$ \\
FEVER & $63(58.3)$ & $15(68.2)$ & $6(30)$ & $0(0)$ \\
DYSPNOEA & $52(48)$ & $9(10.9)$ & $9(45)$ & $0(0)$
\end{tabular}




\begin{tabular}{|c|c|c|c|c|}
\hline DIARRHEA & $18(16.7)$ & $7(31.8)$ & $0(0)$ & $0(0)$ \\
\hline ANOSMIA/AGEUSIA & $17(15.7)$ & $1(4.5)$ & $0(0)$ & $0(0)$ \\
\hline NAUSEA & $17(15.7)$ & $6(27.3)$ & $1(5)$ & $0(0)$ \\
\hline \multicolumn{5}{|l|}{ COMPLICATIONS DURING HOSPITALIZATION (N, \%) } \\
\hline ACUTE RESPIRATORY DISTRESS SYNDROME & $21(19.4)$ & $13(59)$ & $2(10)$ & $0(0)$ \\
\hline ACUTE KIDNEY INJURY & $17(15.7)$ & $12(54.5)$ & $4(20)$ & $0(0)$ \\
\hline ACUTE CARDIAC EVENT & $2(1.9)$ & $1(4.5)$ & $0(0)$ & $0(0)$ \\
\hline ACUTE PULMONARY EMBOLISM & $4(3.7)$ & $0(0)$ & $0(0)$ & $0(0)$ \\
\hline SHOCK & $3(2.8)$ & $3(13.6)$ & $1(5)$ & $0(0)$ \\
\hline PANCREATITIS & $2(1.9)$ & $0(0)$ & $0(0)$ & $0(0)$ \\
\hline VENOUS THROMBOEMBOLISM & $3(2.8)$ & $1(4.5)$ & $1(5)$ & $0(0)$ \\
\hline STROKE & $1(0.9)$ & $0(0)$ & $1(5)$ & $0(0)$ \\
\hline SECONDARY INFECTIONS $(N, \%)$ & $54(50)$ & $19(86.4)$ & $10(50)$ & $0(0)$ \\
\hline $\operatorname{ANTIBIOTICS}(N, \%)$ & $54(50)$ & $19(86.4)$ & $17(85)$ & $0(0)$ \\
\hline OXYGEN SUPPORT WITHOUT VENTILATION (N, \%) & $44(40.7)$ & $3(13.6)$ & $6(30)$ & $0(0)$ \\
\hline VENTILATION SUPPORT $(N, \%)$ & $24(22.2)$ & $14(63.6)$ & $5(25)$ & $0(0)$ \\
\hline ARTIFICIAL NUTRITION (N, \%) & $17(16)$ & $12(54.5)$ & $5(25)$ & $0(0)$ \\
\hline INTENSIVE CARE $(N, \%)$ & $30(27.8)$ & $15(68.2)$ & $5(25)$ & $0(0)$ \\
\hline $\operatorname{IMMUNOSUPPRESSION}(N, \%)$ & $40(37)$ & $5(22.7)$ & $3(15)$ & $2(7.7)$ \\
\hline SPECIFIC CANCER THERAPY $(N, \%)$ & $5(4.6)$ & $2(9)$ & $2(10)$ & $0(0)$ \\
\hline \multicolumn{5}{|l|}{ SPECIFIC SARS-COV-2-TREATMENT $(N, \%)$} \\
\hline REMDESIVIR & $15(13.9)$ & $4(18.2)$ & $0(0)$ & $0(0)$ \\
\hline CONVALESCENT PLASMA & $5(4.6)$ & $1(4.5)$ & $0(0)$ & $0(0)$ \\
\hline $\begin{array}{l}\text { INTRAVENOUS } \\
\text { IMMUNOGLOBULINS }\end{array}$ & $1(0.9)$ & $0(0)$ & $0(0)$ & $0(0)$ \\
\hline BARICITINIB & $1(0.9)$ & $0(0)$ & $0(0)$ & $0(0)$ \\
\hline
\end{tabular}

Table 1 Demographic and Clinical Characteristics of the Study Population

\section{Patient Recruitment and Sampling}

Acquisition of patients was conducted at the university hospital Klinikum rechts der Isar, Technical University Munich, Germany. COVID-19 patients, post COVID-19 patients and SC were prospectively recruited between April 2020 to July 2020 (first COVID-19 wave in Germany) and August 2020 to December 2020 (second COVID-19 wave in Germany), whereas the AC group was prospectively recruited between August 2019 and October 2020. Because these were control patients in an intestinal microbiome-only study, saliva was not obtained (Figure 1 A). Stool, saliva and blood samples were collected at least once per week during the inpatient stay. To ensure follow-up and bio-sample collection after discharge, patients were invited to follow-up visits. SARS-CoV-2 infection was confirmed by quantitative reverse transcription PCR (RT-qPCR), performed on nasopharyngeal swabs. For the post COVID-19 patients the first stool sample was collected on average 30 days after the negative PCR. In the AC group, stool samples were collected either before or 6 weeks after bowel preparation for colonoscopy. To characterize the disease activity, laboratory parameters and data regarding 
112 fraction of inspired oxygen (FiO2), ventilation mode, diet, intensive or normal ward and

113 antibiotic use were collected at each time point of stool or saliva sampling.

\section{Classifications}

116 Patients with COVID-19 or post COVID-19 were classified into 3 groups based on the WHO

117 ordinal scale for clinical improvement for hospitalized patients with COVID-19[24], which has

118 been used in other COVID-19 studies[25]: (i) mild disease, composed of patients with no oxygen therapy (score 3) or oxygen by mask or nasal prongs (score 4); (ii) severe disease, including patients requiring non-invasive ventilation or high-flow oxygen (score 5), intubation and mechanical ventilation (score 6) or ventilation and additional organ support (score 7), and (iii) fatal disease (death, score 8). Ventilation mode during inpatient stay was divided in two groups: (i) Oxygen via nasal prongs, and (ii) mechanically ventilated either pressure controlled (PC) or pressure assisted (PA) and tracheostomy (TS) after long period of intubation. either fed normally or with formulated food via gastric tube in combination with or without parenteral nutrition (summarized in tube feeding).

\section{Ethical Approval}

131 All patients provided written informed consent. The study was conducted in accordance with

132 the declaration of Helsinki and approved by the ethics committee of the Technical University

133 Hospital of Munich (221/20 S-SR).

\section{Sample Preparation and 16S rRNA Gene Sequencing}

136 Faecal and saliva samples were stored in a solution to stabilize DNA (MaGix PBI, Microbiomix

$137 \mathrm{GmbH}$, Regensburg Germany). Sample preparation and paired-end sequencing was performed 
on an Illumina MiSeq targeting the V3V4 region of the 16S rRNA gene. Detailed description

139 of the methods is published[26]. Raw FASTQ files were processed using the NGSToolkit

140 (https://github.com/TUM-Core-Facility-Microbiome/ngstoolkit) based on USEARCH 11[27]

141 to generate denoised zero-radiation operational-taxonomic units (zOTUs).

\section{Statistical Analysis}

144 Differences in relative abundance of taxa and/or zOTUs were determined by Kruskal-Wallis

145 Rank Sum test for multiple groups and Wilcoxon Rank Sum test for pairwise comparison.

146 Differences in prevalence were determined by a non-linear Fisher Exact test. Spearman

147 correlation was calculated for associations and continuous variables.

148 Similarity between samples was estimated based on a distance matrix using generalized

149 UniFrac. Significance between groups, effect modifier, and confounder were determined by a

150 permutational multivariate analysis of variances (adonis function of the R-package vegan).

151 For all analyses, p-values were corrected for multiple testing according to Benjamini-Hochberg

152 correction.

153 The explained variation of co-variables was determined by calculating $\mathrm{R}^{2}$ values and were

154 considered as significant with a $\mathrm{p}$-value $\leq 0.05$. A random forest model was used to classify

155 binary outcome variables based on microbial composition with a 5-fold cross validation by

156 using randomForest from the $\mathrm{R}$ package randomForest v4.6-14. To receive a robust and

157 generalizable classification model, the machine-learning algorithm was applied 100-times

158 iteratively. Based on out-of-bag error rates and Gini index, the most important features were

159 selected for each iteration using $r f c v$ from the R package randomForest v4.6-14. Features, which

160 appeared in all 100 random forest models, were considered as classification features for the

161 final model. A generalized linear model for binomial distribution and binary outcome (logit)

162 was generated using the previously selected features. 


\section{RESULTS}

165

166

167

168

169

170

171

172

173

174

175

176

177

\section{Association of SARS-CoV-2 Status with the Gut Microbiota}

Analysis of the gut microbiota was performed on 251 stool samples $(n=251)$ from 144 patients ( $\mathrm{N}=144)$, of which were 86 COVID-19 patients ( $\mathrm{n}=150$ samples), 21 post COVID-19 patients $(\mathrm{n}=60), 11 \mathrm{SC}(\mathrm{n}=15)$ and $26 \mathrm{AC}(\mathrm{n}=26)$ (Figure 1 A). No bias due to sampling phases was observed allowing a combined analysis of the two COVID-19 waves.

Phylogenetic analysis of patient's microbial profile showed no cluster according to SARS-CoV2 status. Nevertheless, some patients were found to have an increased relative abundance of Proteobacteria which was mainly observed with COVID-19 and post COVID-19 samples (Figure 1 C). The analysis of alpha-diversity revealed a not normally distributed number of observed species and bacterial diversity (Figure 1 B). The number of observed species was reduced in active COVID-19 (richness $133 \pm 90$ ) and post COVID-19 patients (richness $103 \pm$ 60) compared to AC (richness $219 \pm 68$ ), and bacterial diversity showed a reduced Shannon effective number in SC (Figure 1 D).

Considering only the first sampling time point (T1) per individual revealed that parameters related to patient's health were important effect modifiers (Figure 1 D). Interestingly, even though the SARS-CoV-2 status alone did not show a clear pattern in the phylogenetic tree, the detection of SARS-CoV-2 in nasopharyngeal swabs significantly influenced the gut microbiota $\left(R^{2}=0.04, p=0.001\right)$, as well as disease related variables, e.g. the disease severity $\left(R^{2}=0.05\right.$, $\mathrm{p}=0.001)$

\section{Evaluation of Confounding Factors}

Although variables known to influence the microbial composition of the gut such as antibiotics or chemotherapy, appeared to be significant influencing factors (Figure 1 D), none of the tested variables were confounders within the analysed cohort (Supplementary Table 2). Particular attention was paid to variables related to hospitalization such as artificial feeding, critical care 
and antibiotic treatment. Since most patients were treated with different groups of antibiotics,

191 we could not elucidate the influence of a specific antibiotic subgroup on the composition of the

192 gut microbiota. Additionally, patients' comorbidities and disease history was tested for

193 confounding, considering type 2 diabetes[28, 29], inflammtory bowel disease[30], cancer, as

194 well as chemotherapy and immunotherapy[31] within 6 months before stool sampling, or bowel

195 resection[32] (Supplementary Table 3). We further tested whether age and gender, specific

196 SARS-CoV-2 treatment (remdesivir, convalescent plasma, intravenous immunoglobulins, or

197 baricitinib), immunosuppressive therapy, or secondary infections introduced bias in the

198 microbial analysis. Of note, critically ill patients with complications, compared to mild courses,

199 were mainly treated at the ICU and received antibiotics (Supplementary Table 4). However, 200 within the cohort none of the above mentioned variables had a confounding effect in the 201 analysis of the microbial composition related to COVID-19.

\section{Disease Severity and Progression Are Related to Altered Gut Microbiota}

204 Disease severity according to WHO ordinal scale for clinical improvement significantly 205 influences the gut bacterial composition of stool samples $(\mathrm{p}=0.001)$ (Figure 2 A). Beta206 diversity clearly demonstrated a shift of bacterial profiles comparing controls with COVID-19 and post COVID-19 patients. Thereby, the bacterial composition of patients with a mild disease was more similar to SC and AC and a more severe disease showed a microbial composition more similar to patients who died due to COVID-19. A number of stool samples clustered 210 independently in patients with severe and fatal COVID-19 disease, as well as a few mild courses 211 and SC (Figure 2 A, left cluster). However, patients with mild disease in this cluster, or SC, 212 showed no similarities for clinical or laboratory parameters with severe cases. None of the AC 213 samples fell within this cluster.

214 Differentiation analysis revealed zOTUs (Supplementary Table 5), which were significantly 215 different between study groups and correlated with markers of inflammation, such as white 
216 blood cells counts (WBC), C-reactive protein (CRP) and procalcitonin (PCT) (Figure 2 B).

217 Here, Clostridium innocuum (zOTU62), Ruthenibacterium lactatiformans (zOTU29), and

218 Alistipes finegoldii (zOTU64) correlated positively with inflammatory markers and continue to

219 show a significantly increased relative abundance or prevalence in patients with a severe disease 220 progression. Negatively correlated zOTUs were significantly decreased in severe and fatal cases 221 of COVID-19 and post COVID-19, such as Faecalibacterium prausnitzii (zOTU20), Blautia 222 luti (zOTU6), Dorea longicatena (zOTU32), Gemmiger formicilis (zOTU30), and Alistipes putredinis (zOTU33). In addition, Fusicatenibacter showed a significantly reduced prevalence in severe cases and was totally absent in patients who died (Figure 2 B). On the other hand, Parabacteroides significantly increased with a more severe disease (Figure 2 B). Betadiversity already showed some accumulation of patients with an increased relative abundance of Protobacteria (Figure 1 B), which was also found to be increased in severe COVID-19 cases (Figure 2 B).

To analyse the associations of the gut microbial composition with COVID-19 severity in greater depth, we defined a subset of patients with certain criteria. This included patients presenting with high inflammatory parameters (CRP $\geq 10 \mathrm{mg} / \mathrm{dl}, \mathrm{PCT} \geq 5 \mathrm{ng} / \mathrm{ml}, \mathrm{WBC} \geq 15 \mathrm{G} / \mathrm{l}), \mathrm{FiO} 2 \geq$ $23240 \%$, requiring mechanical ventilation (PC, PA, TS), were treated at the ICU, and had at least one complication. In addition, WHO disease severity was set to $\geq 6$. Overall, 15 male patients 234 met the criteria (COVID-19, $\mathrm{N}=8$; post COVID-19, $\mathrm{N}=7$ ) and all of them died, 13 due to 235 acute respiratory distress syndrome (ARDS) and 2 of them due to cerebral haemorrhage. 236 Stratification according to disease severity showed that the microbial profile of severe and fatal 237 cases clustered together. These profiles were mainly dominated by an increased relative 238 abundance of Parabacteroides, Lachnoclostridium, and a reduced relative abundance of 239 Blautia, Faecalibacterium, and Ruminococcus (Figure 2 C), which were shown to be 240 underrepresented in COVID-19[9]. There were no significant differences in the bacterial 241 composition between COVID-19 or post COVID-19 patients. Interestingly, AC showed a 
242 higher abundance of Coprococcus, previously demonstrated to be associated with non COVID-

24319 patients[10], and Roseburia, which were reported to be more prevalent in healthy individuals 244 compared to COVID-19 patients[9].

\section{Microbial Analysis of Saliva Samples}

247 Alterations in the oral microbiome have previously been associated with COVID-19 and 248 suggested as a diagnostic marker[33]. To comprehensively analyse the oro-intestinal bacterial 249 composition, saliva samples were collected in addition to fecal samples (Figure 1 A, 250 Supplementary Table 6). In total, 160 saliva samples from 117 patients were analysed (COVID-19, $\mathrm{N}=87, \mathrm{n}=117$; post COVID-19, $\mathrm{N}=13, \mathrm{n}=24 ; \mathrm{SC}, \mathrm{N}=17, \mathrm{n}=19$ ). Taxonomic differences on phyla level are minor with a reduced relative abundance of Firmicutes in COVID-19 compared to post COVID-19 and SC. Post COVID-19 showed an increased relative abundance of Proteobacteria and a reduction in Actinobacteria. Compared to SC, post COVID19 and COVID-19 had an increased abundance of Fusobacteria (Supplementary Figure 1 A).

Overall, microbial composition between the groups showed no significant differences

(Supplementary Figure 1 B). Interestingly, in accordance with our findings regarding the gut bacteria, stratification of patients according to disease severity showed a significant difference in the composition of the oral microbiome $(\mathrm{p}=0.003)$ (Supplementary Figure $\mathbf{1} \mathbf{C})$ as well as significant variations according to the number of complications $(p=0.001)$ (Supplementary

Figure 1 D). However, a random forest model failed to predict mortality in the setting of COVID-19-associated hospitalization for saliva samples.

\section{Alterations of the Gut Microbiota Correlate with Number and Type of Complications}

265 Following the association between severity and changes in the gut microbiota, we further 266 investigated whether microbial changes were found in terms of type and number of 267 complications in COVID-19 and post COVID-19 patients and SC. A maximum of three 
complications per patient were observed. Stratifying patients according to the number of complications revealed a significant distinction between patients with no complications and patients with one or more complications, with a shift in their bacterial profile according to the number of complications ( $\mathrm{p}=0.002$ ) (Figure 3 A). Furthermore, alpha-diversity showed that the abundance of gut bacteria decreased with the number of complications (Figure $3 \mathbf{B}$ ). Interestingly, F. prausnitzii was found to be reduced with an increased number of complications and absent in patients with three complications (Figure 3 B). Consistent with the findings regarding disease severity (Figure 2 B), Parabacteroides was increased in patients with a more complicated course (Figure 3 B).

Some complications showed overlapping bacteria, which were significantly different in their relative abundance compared to patients without the corresponding complication. Patients who developed ARDS, AKI, or had hemodialysis, revealed a significantly reduced gut bacterial richness as well as Shannon effective number, which was also seen in patients with an acute was significantly reduced in patients with ARDS, AKI, hemodialysis, and acute cardiac events and furthermore negatively associated with mortality. Blautia was reduced for most complications except in patients with VTE/PE or AKI. Parabacteroides, on the other hand, was increased in patients with ARDS and hemodialysis and showed a positive association with mortality. Multivariate permutational analyses showed that AKI had the greatest influence on microbial changes, followed by ARDS, acute cardiac events and VTE. However, pancreatitis and stroke were not significantly contributing to microbial differences (Figure 3 D).

\section{A Stable Gut Bacterial Composition is Correlated with A Favourable Disease Progression}

292 During this study, 39 patients (COVID-19, post COVID-19, and SC) provided more than one 
generalized UniFrac distances the stability of the microbial composition of the gut was

295 determined (Figure 4 A). On average, the intra-individual distance was $0.33 \pm 0.09$. The microbial composition was equally dynamic between COVID-19, post-COVID-19, and SC. previous results (Figure $3 \mathbf{A}$ ) that the onset of complications during inpatient stay significantly

300 correlated with an altered bacterial composition $(\mathrm{p}=0.001)($ Figure 4 B). Even though the intra-individual distance showed no obvious grouping based on SARS-CoV-2 status, a cluster patients without any complication had a more stable microbial composition compared to patients with complications (Figure 4 C). Analysis of the intra-individual microbial stability accounting for varying conditions, demonstrated the significance of environmental factors in addition to the disease state. In the context of intra-individual examination of the bacterial profiles over time, disease progression could be tracked using inflammation markers (CRP, PCT, WBC) and oxygen demand (FiO2) at the time of each stool sample. Thus, we defined a group of COVID-19 and post COVID-19 patients with severe progression. Criteria for a severe progression had to be met at least for one sampling time point $(\mathrm{CRP} \geq 10 \mathrm{mg} / \mathrm{dl}, \mathrm{PCT} \geq 5 \mathrm{ng} / \mathrm{ml}$, $\mathrm{WBC} \geq 15 \mathrm{G} / 1, \mathrm{FiO} 2 \geq 40 \%$ ) and we compared this group (S-prog, $\mathrm{N}=44$ ) with patients not meeting these criteria (NS-prog, $\mathrm{N}=62$ ). Indeed, the bacterial composition of S-prog

313 significantly differed from NS-prog (Figure 4 D).

314 Additionally, machine learning was applied to differentiate between S-prog and NS-prog. 315 Towards this end, a random forest model was trained on COVID-19 patients in a 10-fold crossvalidated nested approach (repeated 100 times). In total, 12 zOTUs were selected as important

317 features: Enterococcus durans (zOTU1), Streptococcus thermophilus (zOTU119, zOTU25), 318 Citrobacter freundii (zOTU137, zOTU76), Holdemania massiliensis (zOTU293), 
(zOTU442), Blautia spp. (zOTU54, zOTU6), Lacticaseibacillus rhamnosus (zOTU924)

321 (Figure 4 D, Supplementary Table 5). The defined signature was overlapping with zOTUs (AUC) of 0.94 to predict mortality during the COVID-19 associated inpatient stay (Figure 4

D). The specificity was verified by applying the signature to other outcomes, e.g. type 2 diabetes $(\mathrm{AUC}=0.76)$ or presence of complications $(\mathrm{AUC}=0.82)$

\section{DISCUSSION}

329 The risk of a severe disease course and complications, including thromboembolism, renal 330 failure, and acute cardiac events is higher for COVID-19 than for influenza[34]. GI symptoms 331 in patients with COVID-19 are associated with an increased disease severity and 332 complications[8] and an exaggerated immune response to the virus is considered to play a 333 crucial role in driving disease progression[15, 16]. It is well known that gut microorganisms

334 influence the systemic immune response of their hosts through multiple crosstalk with immune cells[35, 36, 37].

336 In our study, we demonstrated that the bacterial composition of the gut in patients with COVID19 disease changes with number and type of complications. Thereby, taxa known for protective and immunosuppressive properties were found to be decreased with an increasing complication

339 rate, whereas rather pathogenic taxa were more prevalent. $F$. prausnitzii, for example, was 340 undetectable in patients with three complications and relatively reduced in patients with AKI,

341 hemodialysis, ARDS, cardiac event and was negatively correlated with mortality. This 342 bacterium has anti-inflammatory properties[38, 39] and was found to have an inverse 343 correlation with disease severity in COVID-19[9, 10]. On the other hand, the relative abundance 344 of the genus Alistipes was increased with the number of complications. In terms of functionality, there is conflicting evidence to the protective or pathogenic potential of Alistipes in various 
346 diseases[40]. In patients with thromboembolic complications the genus Tyzzerella was the only

347 significantly elevated bacterium. Interestingly, Tyzzerella was previously shown to be 348 associated with an increased risk of cardiovascular diseases[41]. Parabacteroides was

349 increased in patients with ARDS and hemodialysis and related to mortality. The associations of 350 individual bacteria with the occurrence of complications suggests a potential role of the gut 351 microbiota in the development of specific complications within COVID-19 and provide 352 additional evidence for an involvement of the gut concerning cardiovascular risk[42] and 353 venous thromboembolism[43, 44].

354 In addition, differences in the bacterial composition were found dependent on the disease severity. While the microbial profile of patients with mild diseases was comparable to controls, severe and fatal cases showed marked differences with respect to protective bacteria. Congruent 357 with previously published studies in other countries[1,9, 10, 45], our results confirm a link between disease severity of COVID-19 and microbiota alterations in a large German cohort. Besides an inverse correlation of $F$. prausnitzii with disease severity of COVID-19[10], Blautia was previously shown to be underrepresented in patients with COVID-19 and was associated 361 with SARS-CoV-2 recovery[9]. Fusicatenibacter was reported to be enriched in non COVID36219 controls[45] and correlated negatively with inflammatory biomarkers in COVID-19 363 patients[46] and Parabacteroides correlated positively with disease severity[9].

364 To more deeply examine the associations of the gut bacteria with COVID-19 progression, we 365 considered functional data, such as FiO2, at each time of stool collection. Thereby, the intra366 individual microbial stability decreased with a higher complication rate. Based on a distinct 367 microbial profile, the individual risk of mortality due to COVID-19 could be estimated. Thus, 368 while disease severity, inflammatory activity, and complication rate were associated with 369 changes in bacterial composition in COVID-19 patients, the impact of SARS-CoV-2 infection 370 appears to be more modest, indicating that the gut plays a role in shaping severe disease 371 progression. 
372 Regarding the microbiota changes in the oral cavity, differences in bacterial composition related

373 to severity and complications were observed, highlighting the importance of the bacterial oro-

374 intestinal axis in COVID-19[33]. However, prediction of mortality was not feasible using

375 bacterial patterns in saliva and the results were less conclusive compared to changes in the gut

376 microbiota.

377 We hypothesize that changes in the microbial composition, especially of the gut, may drive 378 disease, possibly via an involvement in the development of complications. A stable bacterial 379 profile during hospitalization could have a favorable impact on disease progression. A healthy 380 and diverse intestinal microbiota should, therefore, be considered in the therapeutic 381 management of COVID-19.

382 Because of the often prolonged hospital stay of inpatients of 24 days on average within our 383 cohort, multiple factors could influence the gut microbiota. These include formulated food, 384 antibiotics, or catabolic metabolism during an ICU stay[47]. Especially in a clinically 385 heterogeneous disease like COVID-19, these factors must be considered in the interpretation of 386 microbiota analysis. For this reason, we carefully reviewed the results for potential 387 confounders, including concomitant diseases and assessable factors associated with 388 hospitalization. In this context, none of the factors examined was found to be a confounder with 389 significant bias concerning our results. Nevertheless, patients with a severe and complicating 390 disease, in contrast to mild cases, were mainly treated at the ICU and given antibiotics 391 (Supplementary Table 4). Thus, it cannot be ruled out that microbiota changes related to the 392 severity and complications are also influenced by the conditions of medical treatment. It further 393 remains unclear whether the changes in microbiota causally influenced the severity of COVID39419 and occurrence of complications, or vice versa.

395 Taken together, our results suggest that the gut and salivary microbiota are associated with the 396 occurrence of individual complications in COVID-19, thereby influencing disease severity. A 397 stable gut bacterial composition during hospitalization is associated with a more favorable 
398 clinical course. Further studies are needed to investigate direct causality between gut bacterial

399 dysbiosis and COVID-19 and to integrate microbial patterns for prognostic and therapeutic

400 purposes in clinical routine.

401

402 
Conflict of Interest: All authors have no conflict of interest to disclose.

404

Acknowledgments: We thank all the health care workers of Klinikum rechts der Isar as well

as the CoMRI team around Christoph Spinner, MD. We are grateful to Angela Sachsenhauser,

Caroline Ziegler and Lukas Mix from the Core Facility Microbiome of the ZIEL Institute for

Food \& Health for outstanding technical support in sample preparation and 16S rRNA gene

amplicon sequencing

Authorship Contributions: David Schult, MD (Conceptualization: Lead; Project

administration: Lead; Supervision: Lead; Investigation: Equal; Methodology: Lead;

Visualization: Supporting; Validation: Equal; Manuscript - writing: Lead, Manuscript - review

\& editing: Lead), Sandra Reitmeier, PhD (Conceptualization: Equal; Project administration:

Plamena Koyumdzhieva, cand. med. (Conceptualization: Equal; Investigation: Lead;

Methodology: Supporting; Visualization: Supporting; Validation: Equal; Manuscript - writing:

Equal, Manuscript - review \& editing: Supporting), Tobias Lahmer, MD, Johanna Erber, MD,

Marina Frolova, Julia Horstmann, Lisa Fricke, Juliane Kager, MD, Katja Steiger, MD, Moritz

Manuscript - review \& editing: Supporting), Ulrike Protzer, MD (Resources: Supporting),

Klaus Neuhaus, PhD (Resources: Equal; Validation: Supporting; Manuscript - review \& 
428 (Conceptualization: Lead; Project administration: Equal; Supervision: Lead; Funding acquisition: Lead; Manuscript - review \& editing: Lead).

Data Availability and Data Transparency Statement: FASTQ files of the 16S rRNA gene (https://www.ncbi.nlm.nih.gov/bioproject/PRJNA756849/).

Keywords: Coronavirus; SARS-CoV-2; Complications, Gut Microbiome; Oral Microbiome

Funding: Internal funds of Technical University of Munich to CoMRI (Cohort study for patients tested positive for SARS-CoV-2), Deutsche Forschungsgesellschaft (DFG) grant 395357507 SFB 1371

Abbreviations: $\mathrm{AB} \mathrm{T} 1=$ Antibiotic therapy at the time of the first stool sampling; $\mathrm{AC}=$ Asymptomatic controls; AKI = Acute kidney injury; AP = Alkaline phosphatase [U/l]; ARDS

$443=$ Acute respiratory distress syndrome; Asympt. = Asymptomatic; COVID-19 = Corona virus 444 disease 2019; CRP = C-reactive protein [mg/dl]; FiO2 = Fraction of inspired oxygen (\%); GGT $445=$ Gamma-Glutamyltransferase [U/l]; GI = Gastrointestinal; $\mathrm{Hb}=$ Hemoglobin $[\mathrm{g} / \mathrm{dl}]$; i.v. = 446 intravenous; IBD = Inflammatory bowel disease; $\mathrm{ICU}=$ Intensive care unit; ICU all $\mathrm{T}=$ 447 Intensive care stay regarding all time points of stool sampling; ICU T1 = Intensive care stay at 448 the time of the first stool sampling; $\mathrm{N}=$ Number of patients; $\mathrm{n}=$ Number of samples; $\mathrm{NA}=$ not 449 available; $\mathrm{PA}=$ Pressure assisted; $\mathrm{PC}=$ Pressure controlled; $\mathrm{PCT}=$ Procalcitonin $[\mathrm{ng} / \mathrm{ml}] ; \mathrm{PE}=$ 450 Pulmonary embolism; Rel. = Relative; S.p. = Status post; SARS-CoV-2 = Severe acute 451 respiratory syndrome coronavirus 2 ; $\mathrm{SC}=$ Symptomatic pneumonia controls; Sig. = Significant; 452 Sympt. $=$ Symptomatic; $\mathrm{T} 1=$ First sampling time point; $\mathrm{T} 2 \mathrm{D}=$ Type 2 diabetes mellitus; $\operatorname{Trp} \mathrm{T}$ 
453 = High-sensitive troponin $\mathrm{T}$ [ng/ml]; TS = Tracheostomy; VTE = Venous thromboembolism;

$454 \mathrm{WBC}=$ White blood cells counts $[\mathrm{G} / \mathrm{l}]$; zOTU $=$ Zero-radiation operational-taxonomic units 455 


\section{References}

4571 Zuo T, Liu Q, Zhang F, Lui GC, Tso EY, Yeoh YK, et al. Depicting SARS-CoV-2

458 faecal viral activity in association with gut microbiota composition in patients with COVID-

459 19. Gut 2021;70:276-84.

4602 Liang W, Feng Z, Rao S, Xiao C, Xue X, Lin Z, et al. Diarrhoea may be

461 underestimated: a missing link in 2019 novel coronavirus. Gut 2020;69:1141-3.

4623 Cheung KS, Hung IFN, Chan PPY, Lung KC, Tso E, Liu R, et al. Gastrointestinal

463 Manifestations of SARS-CoV-2 Infection and Virus Load in Fecal Samples From a Hong

464 Kong Cohort: Systematic Review and Meta-analysis. Gastroenterology 2020;159:81-95.

4654 Wiersinga WJ, Rhodes A, Cheng AC, Peacock SJ, Prescott HC. Pathophysiology,

466 Transmission, Diagnosis, and Treatment of Coronavirus Disease 2019 (COVID-19): A

467 Review. JAMA 2020;324:782-93.

4685 Mao R, Qiu Y, He JS, Tan JY, Li XH, Liang J, et al. Manifestations and prognosis of

469 gastrointestinal and liver involvement in patients with COVID-19: a systematic review and

470 meta-analysis. Lancet Gastroenterol Hepatol 2020;5:667-78.

4716 Lamers MM, Beumer J, van der Vaart J, Knoops K, Puschhof J, Breugem TI, et al.

472 SARS-CoV-2 productively infects human gut enterocytes. Science 2020;369:50-4.

4737 Xu Y, Li X, Zhu B, Liang H, Fang C, Gong Y, et al. Characteristics of pediatric

474 SARS-CoV-2 infection and potential evidence for persistent fecal viral shedding. Nat Med

475 2020;26:502-5.

4768 Jin X, Lian JS, Hu JH, Gao J, Zheng L, Zhang YM, et al. Epidemiological, clinical

477 and virological characteristics of 74 cases of coronavirus-infected disease 2019 (COVID-19)

478 with gastrointestinal symptoms. Gut 2020;69:1002-9.

4799 Zuo T, Zhang F, Lui GCY, Yeoh YK, Li AYL, Zhan H, et al. Alterations in Gut

480 Microbiota of Patients With COVID-19 During Time of Hospitalization. Gastroenterology

$481 \quad 2020 ; \mathbf{1 5 9}: 944-55$ e8.

48210 Yeoh YK, Zuo T, Lui GC, Zhang F, Liu Q, Li AY, et al. Gut microbiota composition

483 reflects disease severity and dysfunctional immune responses in patients with COVID-19. Gut

484 2021;70:698-706.

48511 Middeldorp S, Coppens M, van Haaps TF, Foppen M, Vlaar AP, Muller MCA, et al.

486 Incidence of venous thromboembolism in hospitalized patients with COVID-19. J Thromb

487 Haemost 2020;18:1995-2002.

48812 Klok FA, Kruip M, van der Meer NJM, Arbous MS, Gommers D, Kant KM, et al.

489 Incidence of thrombotic complications in critically ill ICU patients with COVID-19. Thromb

$490 \quad$ Res 2020;191:145-7.

49113 Alhazzani W, Moller MH, Arabi YM, Loeb M, Gong MN, Fan E, et al. Surviving

492 Sepsis Campaign: guidelines on the management of critically ill adults with Coronavirus

493 Disease 2019 (COVID-19). Intensive Care Med 2020;46:854-87.

49414 Legrand M, Bell S, Forni L, Joannidis M, Koyner JL, Liu K, et al. Pathophysiology of

495 COVID-19-associated acute kidney injury. Nat Rev Nephrol 2021.

$49615 \quad$ Brodin P. Immune determinants of COVID-19 disease presentation and severity. Nat

497 Med 2021;27:28-33.

49816 Hu B, Guo H, Zhou P, Shi ZL. Characteristics of SARS-CoV-2 and COVID-19. Nat

499 Rev Microbiol 2021;19:141-54.

50017 Kosiewicz MM, Zirnheld AL, Alard P. Gut microbiota, immunity, and disease: a

501 complex relationship. Front Microbiol 2011;2:180.

50218 Schirmer M, Smeekens SP, Vlamakis H, Jaeger M, Oosting M, Franzosa EA, et al.

503 Linking the Human Gut Microbiome to Inflammatory Cytokine Production Capacity. Cell

504 2016;167:1125-36 e8. 

Environment dominates over host genetics in shaping human gut microbiota. Nature 2018;555:210-5.

50820 Kau AL, Ahern PP, Griffin NW, Goodman AL, Gordon JI. Human nutrition, the gut microbiome and the immune system. Nature 2011;474:327-36. 21 Yatsunenko T, Rey FE, Manary MJ, Trehan I, Dominguez-Bello MG, Contreras M, et 22 Mueller S, Saunier K, Hanisch C, Norin E, Alm L, Midtvedt T, et al. Differences in fecal microbiota in different European study populations in relation to age, gender, and country: a cross-sectional study. Appl Environ Microbiol 2006;72:1027-33.

23 McDonald D, Ackermann G, Khailova L, Baird C, Heyland D, Kozar R, et al. Extreme Dysbiosis of the Microbiome in Critical Illness. mSphere 2016;1.

24 World Health Organization R\&D Blueprint. COVID-19 Therapeutic Trial Synopsis, http://www.who.int/blueprint/priority-diseases/key-action/novel-coronavirus/en/ (Accessed August 11, 2021).

25 Cao B, Wang Y, Wen D, Liu W, Wang J, Fan G, et al. A Trial of Lopinavir-Ritonavir in Adults Hospitalized with Severe Covid-19. N Engl J Med 2020;382:1787-99.

26 Reitmeier S, Kiessling S, Neuhaus K, Haller D. Comparing Circadian Rhythmicity in the Human Gut Microbiome. STAR Protoc 2020;1:100148.

27 Edgar RC, Flyvbjerg H. Error filtering, pair assembly and error correction for nextgeneration sequencing reads. Bioinformatics 2015;31:3476-82.

28 Reitmeier S, Kiessling S, Clavel T, List M, Almeida EL, Ghosh TS, et al. Arrhythmic Gut Microbiome Signatures Predict Risk of Type 2 Diabetes. Cell Host Microbe 2020;28:25872 e6.

29 Qin J, Li Y, Cai Z, Li S, Zhu J, Zhang F, et al. A metagenome-wide association study of gut microbiota in type 2 diabetes. Nature 2012;490:55-60.

30 Franzosa EA, Sirota-Madi A, Avila-Pacheco J, Fornelos N, Haiser HJ, Reinker S, et al. Gut microbiome structure and metabolic activity in inflammatory bowel disease. Nat Microbiol 2019;4:293-305.

31 Gopalakrishnan V, Helmink BA, Spencer CN, Reuben A, Wargo JA. The Influence of the Gut Microbiome on Cancer, Immunity, and Cancer Immunotherapy. Cancer Cell 2018;33:570-80.

32 Agnes A, Puccioni C, D'Ugo D, Gasbarrini A, Biondi A, Persiani R. The gut microbiota and colorectal surgery outcomes: facts or hype? A narrative review. BMC Surg 2021;21:83.

33 Ren Z, Wang H, Cui G, Lu H, Wang L, Luo H, et al. Alterations in the human oral and gut microbiomes and lipidomics in COVID-19. Gut 2021;70:1253-65.

34 Xie Y, Bowe B, Maddukuri G, Al-Aly Z. Comparative evaluation of clinical manifestations and risk of death in patients admitted to hospital with covid-19 and seasonal influenza: cohort study. BMJ 2020;371:m4677.

35 Atarashi K, Tanoue T, Ando M, Kamada N, Nagano Y, Narushima S, et al. Th17 Cell Induction by Adhesion of Microbes to Intestinal Epithelial Cells. Cell 2015;163:367-80.

36 Honda K, Littman DR. The microbiota in adaptive immune homeostasis and disease. Nature 2016;535:75-84.

37 Mortha A, Chudnovskiy A, Hashimoto D, Bogunovic M, Spencer SP, Belkaid Y, et al. Microbiota-dependent crosstalk between macrophages and ILC3 promotes intestinal homeostasis. Science 2014;343:1249288.

38 Sokol H, Pigneur B, Watterlot L, Lakhdari O, Bermudez-Humaran LG, Gratadoux JJ, et al. Faecalibacterium prausnitzii is an anti-inflammatory commensal bacterium identified by gut microbiota analysis of Crohn disease patients. Proc Natl Acad Sci U S A 2008;105:167316. 
55639 Lopez-Siles M, Duncan SH, Garcia-Gil LJ, Martinez-Medina M. Faecalibacterium 557 prausnitzii: from microbiology to diagnostics and prognostics. ISME J 2017; 11:841-52.

55840 Parker BJ, Wearsch PA, Veloo ACM, Rodriguez-Palacios A. The Genus Alistipes:

559 Gut Bacteria With Emerging Implications to Inflammation, Cancer, and Mental Health. Front 560 Immunol 2020;11:906.

56141 Kelly TN, Bazzano LA, Ajami NJ, He H, Zhao J, Petrosino JF, et al. Gut Microbiome 562 Associates With Lifetime Cardiovascular Disease Risk Profile Among Bogalusa Heart Study 563 Participants. Circ Res 2016;119:956-64.

$56442 \quad$ Wang Z, Klipfell E, Bennett BJ, Koeth R, Levison BS, Dugar B, et al. Gut flora

565 metabolism of phosphatidylcholine promotes cardiovascular disease. Nature 2011;472:57-63.

56643 Liu Y, Dai M. Trimethylamine N-Oxide Generated by the Gut Microbiota Is

567 Associated with Vascular Inflammation: New Insights into Atherosclerosis. Mediators

568 Inflamm 2020;2020:4634172.

56944 Roberts AB, Gu X, Buffa JA, Hurd AG, Wang Z, Zhu W, et al. Development of a gut

570 microbe-targeted nonlethal therapeutic to inhibit thrombosis potential. Nat Med

$571 \quad 2018 ; 24: 1407-17$.

57245 Newsome RC, Gauthier J, Hernandez MC, Abraham GE, Robinson TO, Williams HB, 573 et al. The gut microbiome of COVID-19 recovered patients returns to uninfected status in a 574 minority-dominated United States cohort. Gut Microbes 2021;13:1-15.

57546 Gu S, Chen Y, Wu Z, Chen Y, Gao H, Lv L, et al. Alterations of the Gut Microbiota in 576 Patients With Coronavirus Disease 2019 or H1N1 Influenza. Clin Infect Dis 2020;71:2669-

57778.

57847 Otani S, Chihade DB, Coopersmith CM. Critical illness and the role of the 579 microbiome. Acute Med Surg 2019;6:91-4. 
Figure and Table Legends

Figure 1 Microbial Composition of the Gut Observed in the Cohort

A Overview of study design. Stool and saliva samples are indicated. B Alpha-diversity of all samples of all patients. Stacked barplots show taxonomic distribution on phyla level. Inner label shows SARS-CoV-2 status and outer label indicates the sampling time phase. D Left, alphadiversity stratified according to SARS-CoV-2 status for all samples of all patients, showing contributing to microbial diversity in all samples. $\mathrm{Y}$-axis shows the $\mathrm{R}^{2}$ value calculated based on Bray-Curtis distance for COVID-19, post COVID-19 and SC.

A MDS plot calculated on generalized UniFrac distance stratifying all patients (samples from the first time point, T1, only) according to disease severity. B Heatmap shows significant different taxa between COVID-19, post COVID-19 and SC patients with a different disease severity in correlation to inflammatory biomarkers. WBC, CRP and PCT. Boxplots show significantly different taxa according to disease severity. Fusicatenibacter shows differences in prevalence $(\mathrm{p}$-value $=0.02)$, the genus Parabacteroides and phylum Protobacteria are generalized UniFrac distances between a subset of COVID-19 and post COVID-19 patients, 603 fulfilling certain criteria of a high inflammatory and severe disease, and AC for the sampling at different. 
608 A MDS plot calculated on generalized UniFrac distance stratifying COVID-19 and post 609 COVID-19 patients, SC and AC (T1) according to the number of complications during 610 hospitalization. B Same samples as in panel A, boxplots show significant differences in alpha611 diversity and relative abundance of taxa. Faecalibacterium shows differences in prevalence (p612 value $=0.0002)$ and relative abundance ( $\mathrm{p}$-value $\leq 0.01)$, Parabacteroides and Alistipes are 613 significantly different in their relative abundance ( $p$-value $\leq 0.01$ ). C Heatmap with taxa found 614 to be significantly different in COVID-19, post COVID-19 and SC patients (T1) and with specific complications. Values are showing the mean relative abundance detected in patients with the complication compared to patients without complication. The color code indicates high

617 (green) or low (white) relative abundance. D Multivariate permutational analysis revealed the 618 importance of complications regarding microbial composition. Barplots are showing the $\mathrm{R}^{2}$ 619 values. Green bars $=$ significant variables $(*, p \leq 0.05, * *, p \leq 0.01 ; * * *, p \leq 0.001)$, grey $=$ non-significant variables.

624 A Intra-individual generalized UniFrac distance sorted by median distance within one patient. Longitudinal sampling of at least two samples per patient with a medium of 3.5 (COVID-19), 4.6 (post COVID-19), and 2.3 (SC) samples per patient. Each box represents one patient. Dashed line shows the mean intra-individual distance over all patients $(\mathrm{N}=39)$. Right color bar 628 shows variables related to hospitalization as indicated by the legend. B MDS plot calculated on generalized UniFrac distances stratifying COVID-19, post COVID-19 and SC patients (all sampling time points) according to number of complications. C Intra-individual generalized

631 UniFrac distances sorted by median distance within one patient of the COVID-19 cohort with 632 a minimum of two samples $(\mathrm{N}=25)$. Each box represents one patient. D MDS plot calculated 633 on generalized UniFrac distance stratifying COVID-19 and post COVID-19 patients (all 
634 sampling time points) for disease progression in severe (S-prog) compared to patients not

635 meeting these criteria (NS-prog). E Individual relative abundance values of random forest

636 selected zOTUs for classification of severe disease progression grouped by SARS-CoV-2

637 status. ROC curve shows differentiation by mortality based on previously determined feature

638 list.

639

640 Supplementary Figure 1 Microbial Profile of Sputum Samples

641 A Upper plot shows the taxonomic distribution based on phyla level over all patients (T1).

642 Stacked barplots represent phyla composition stratified by SARS-CoV-2 status. B-D MDS plot

643 calculated on generalized UniFrac distance stratifying at T1 according to B SARS-CoV-2

644 status, C disease severity, D number of complications during hospitalization.

645

Table 1 Demographic and Clinical Characteristics of the Study Population

647

Supplementary Table 1 Classification of the Antibiotic Therapy given to Patients during

their Inpatient Stay

650

Supplementary Table 2 Results of Confounding Analysis

Supplementary Table 3 Distribution of Factors with Possible Impact on the Gut

Microbiota

655

Supplementary Table 4 Antibiotics and Intensive Care According to COVID-19 Severity 
661 Supplementary Table 6 Taxonomic Classification of zOTUs in Saliva Samples 
bioRxiv preprint doi: https://doi.org/10.1101/2021.10.08.463613; this version posted October 9, 2021. The copyright holder for this preprint (which was not certified by peer review) is the author/funder. All rights reserved. No reuse allowed without permission.

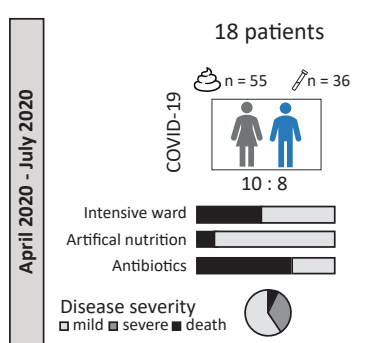

90 patients

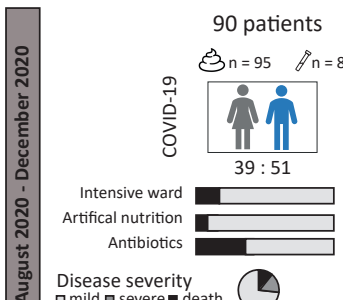

Disease severity
a mild $\mathrm{a}$ severe death

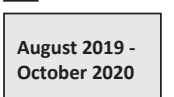

Asympt. controls
26 individuals
है $n=26$

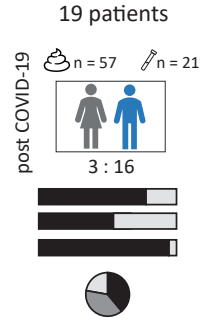

3 patients

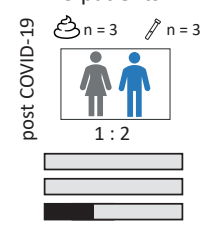

$(1$

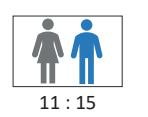

10 patients

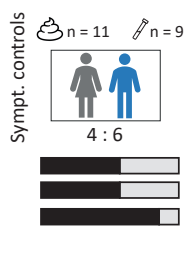

10 patients

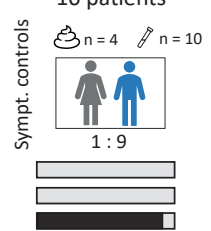

Age \& gender matched

- No antiobiotics \& no chemotherapy
Taxonomic classification phyla level

Actinobacteria

Bacteroidetes
Firmicutes

Fusobacteria

Proteobacteria

Verrucomicrobiota

SARS-CoV-2 status

COVID-19 ( $\mathrm{N}=86, \mathrm{n}=150)$

Post COVID-19 $(\mathrm{N}=21, \mathrm{n}=60)$

Sympt. controls $(\mathrm{N}=11, \mathrm{n}=15)$

Asympt. controls $(\mathrm{N}=26)$

Sampling period

First COVID-19 wave in Germany
(April 2020 - July 2020)

Second COVID-19 wave in Germany
(August 2020 - December 2020)

Asympt. controls
(August 2019 - Oc

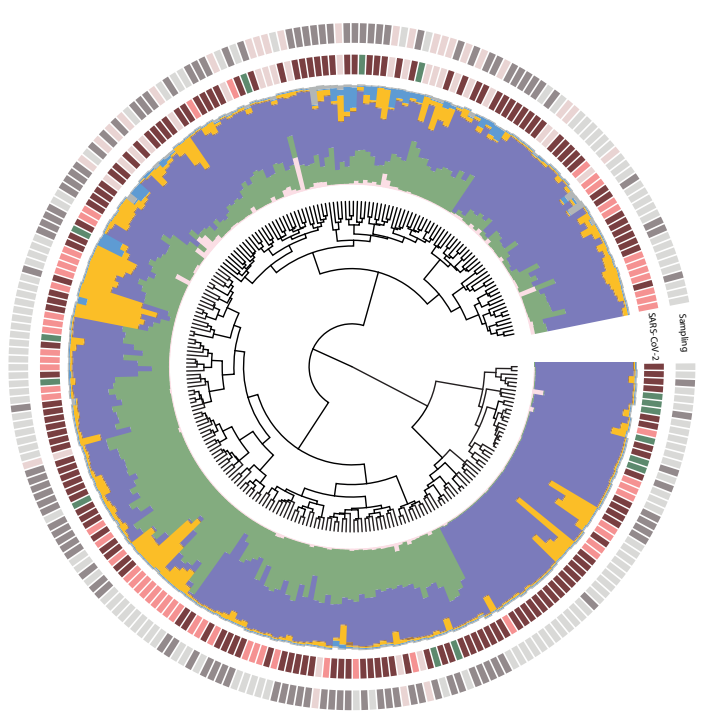

b

Number of observed zOTUs

Bacterial diversity
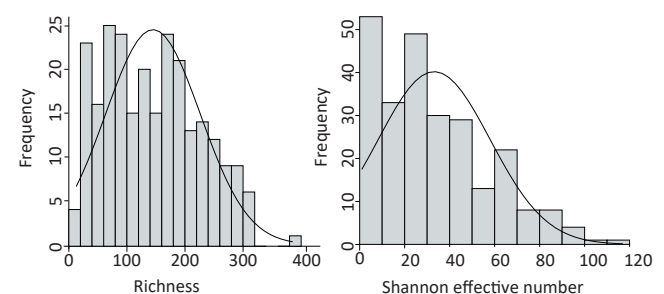

d

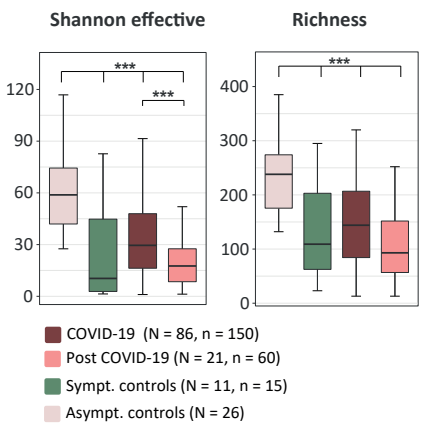

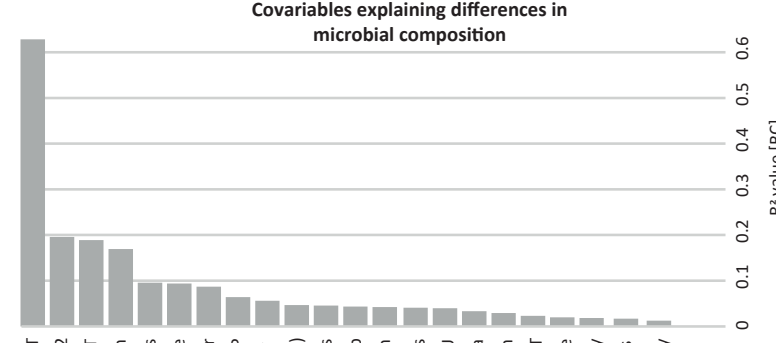

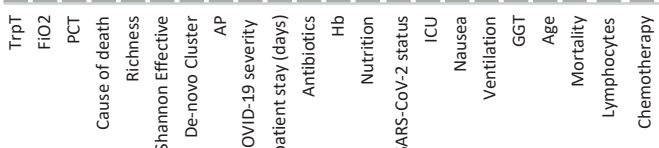


bioRxiv preprint doi: https://doi.org/10.1101/2021.10.08.463613; this version posted October 9, 2021. The copyright holder for this preprint (which was not certified by peer review) is the author/funder. All rights reserved. No reuse allowed without permission.

Asympt. controls $(\mathrm{N}=26)$

Sympt. controls $(\mathrm{N}=11)$

Mild disease $(\mathrm{N}=68)$

$d=0.1$

Severe disease $(N=22)$ p-value $=0.001$

- Death $(\mathrm{N}=17)$
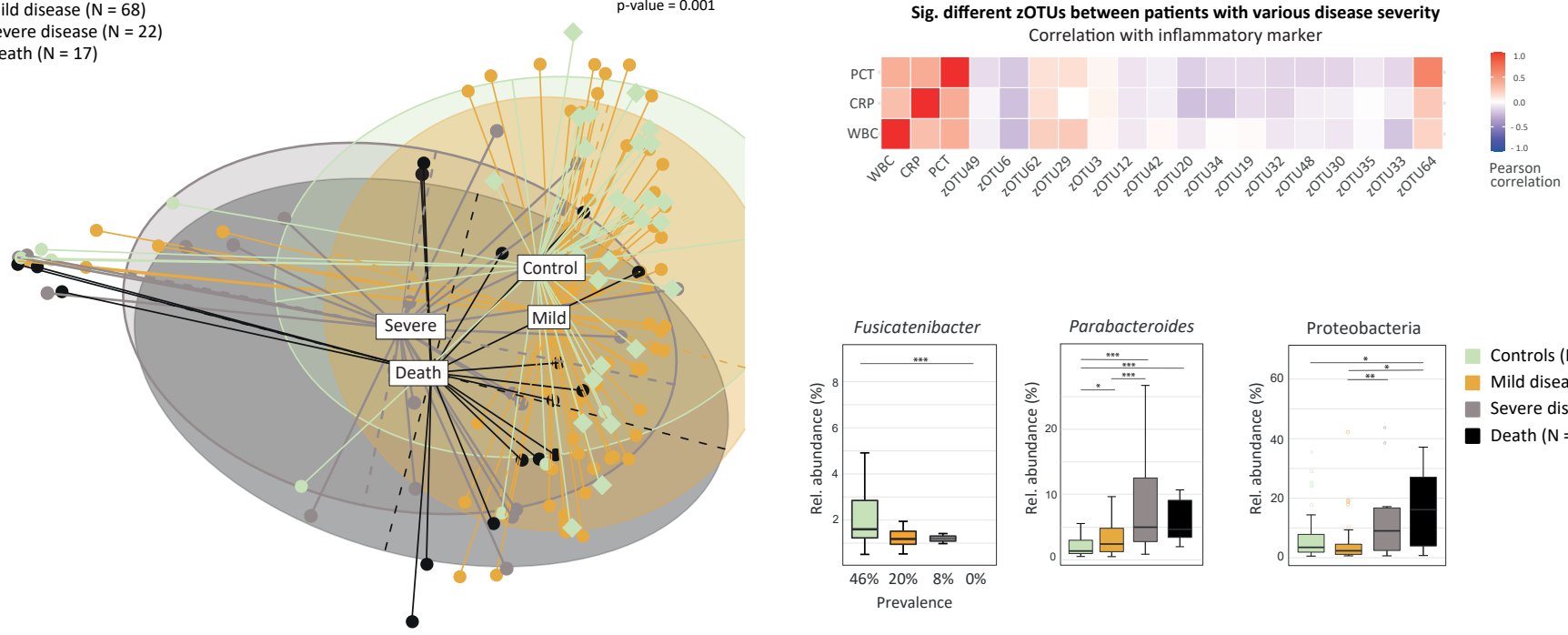

Sig. different genera

\begin{tabular}{l}
\hline Bacteroides \\
Barnesiella \\
Blautia \\
Coprococcus \\
Faecalibacterium \\
Fusciatenibacter \\
Lachnoclostridium \\
Parabacteroides \\
Roseburia
\end{tabular}
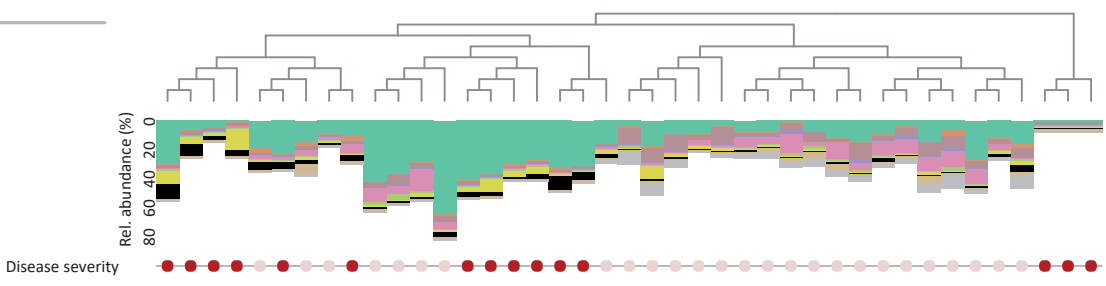

SARS-CoV-2 status

Disease severity and SARS-CoV-2 status

WHO classification 7 or $8(\mathrm{~N}=15)$
Asympt. control $(\mathrm{N}=26)$

$\operatorname{COVID-19}(\mathrm{N}=8)$

Post COVID-19 ( $\mathrm{N}=7$ )

Controls $(\mathrm{N}=37)$

Mild disease ( $\mathrm{N}=68$ )

Severe disease $(\mathrm{N}=22)$

Death $(N=17)$ 
bioRxiv preprint doi: https://doi org/10.1101/2021.10.08.463613; this version posted October 9,2021 . The copyright holder for this preprint (which was not certified by peer review) is the author/funder. All rights reserved. No reuse allowed without permission.

a

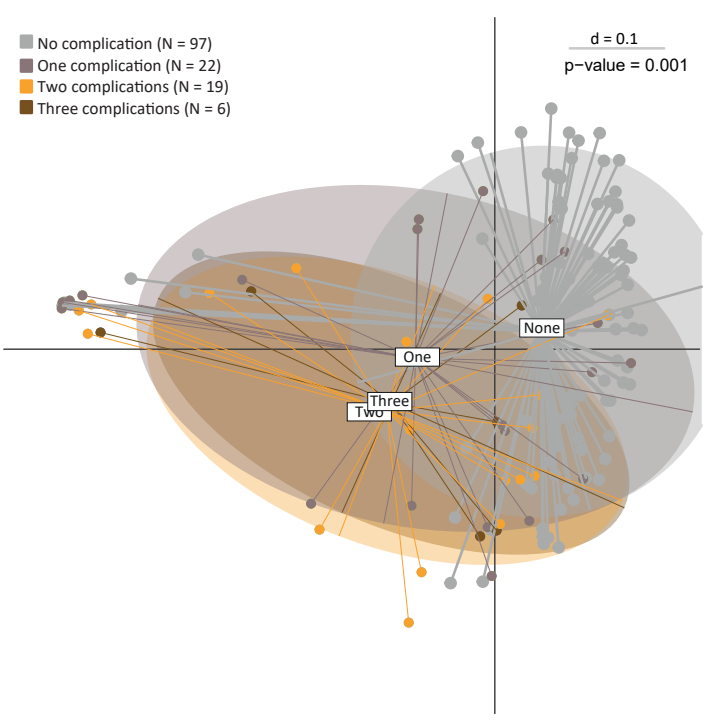

C

Sig. differenences between complications
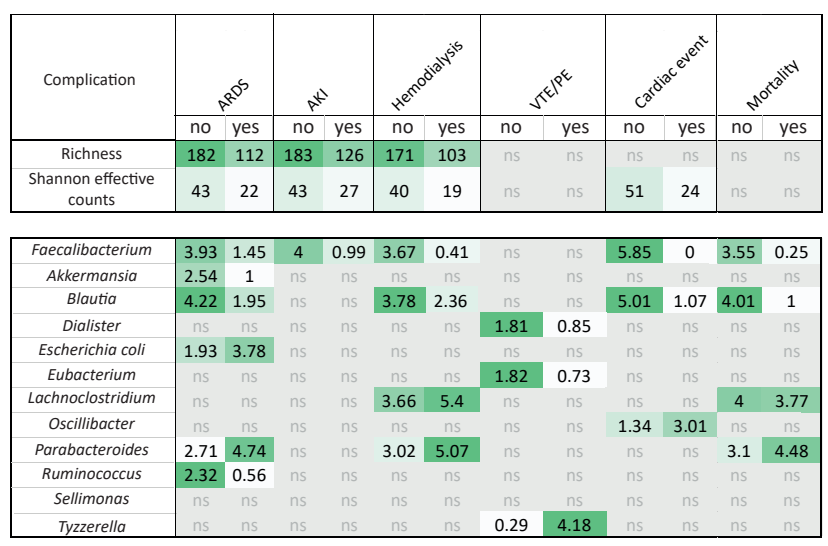

\begin{tabular}{|l|cccccc|c|c|c|c|c|c|}
\hline zOTU12 & ns & ns & ns & ns & ns & ns & 3.33 & 0 & ns & ns & ns & ns \\
\hline zOTU18 & ns & ns & ns & ns & ns & ns & 2.38 & 0.67 & ns & ns & ns & ns \\
\hline zOTU19 & ns & ns & ns & ns & 1.01 & 0 & ns & ns & ns & ns & ns & ns \\
\hline zOTU20 & ns & n5 & ns & ns & 1.01 & 0 & ns & ns & ns & ns & ns & ns \\
\hline zOTU3 & 1.93 & 3.78 & ns & ns & ns & ns & ns & ns & ns & ns & ns & ns \\
\hline zOTU30 & ns & ns & 0 & 1.01 & 0.9 & 0 & ns & ns & ns & ns & ns & ns \\
\hline zOTU36 & ns & ns & ns & ns & ns & ns & ns & ns & 1.25 & 2.83 & ns & ns \\
\hline zOTU6 & 1.61 & 0 & ns & ns & ns & ns & ns & ns & ns & ns & 1.38 & 0.17 \\
\hline
\end{tabular}

b
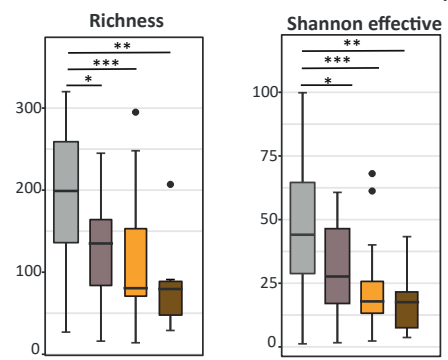

No complication $(\mathrm{N}=97)$ One complication $(\mathrm{N}=22)$ Three complications $(N=6)$
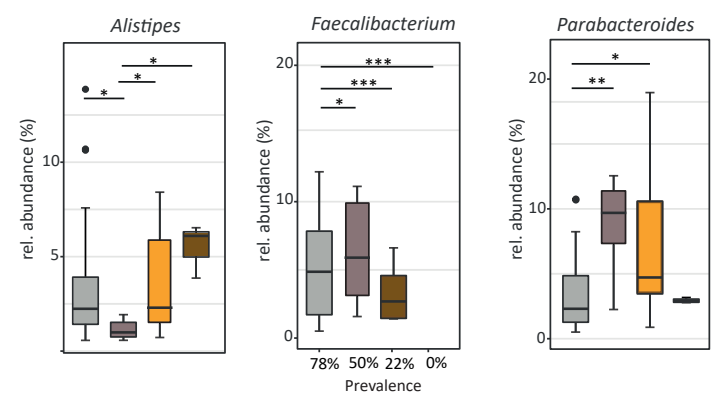

d

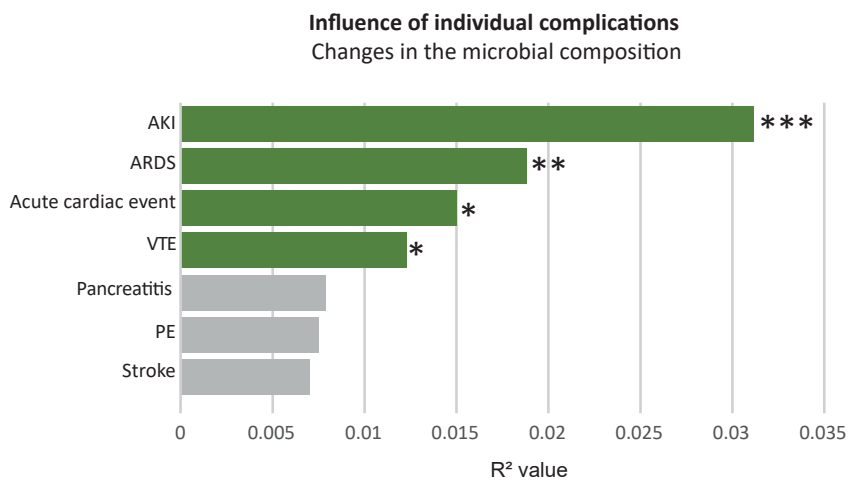


bioRxiv preprint doi: https://doi.org/10.1101/2021.10.08.463613; this version posted October 9,2021 . The copyright holder for this preprint a (which was not certified by peer review) is the author/funder. All rights reserved. No reuse allowed without

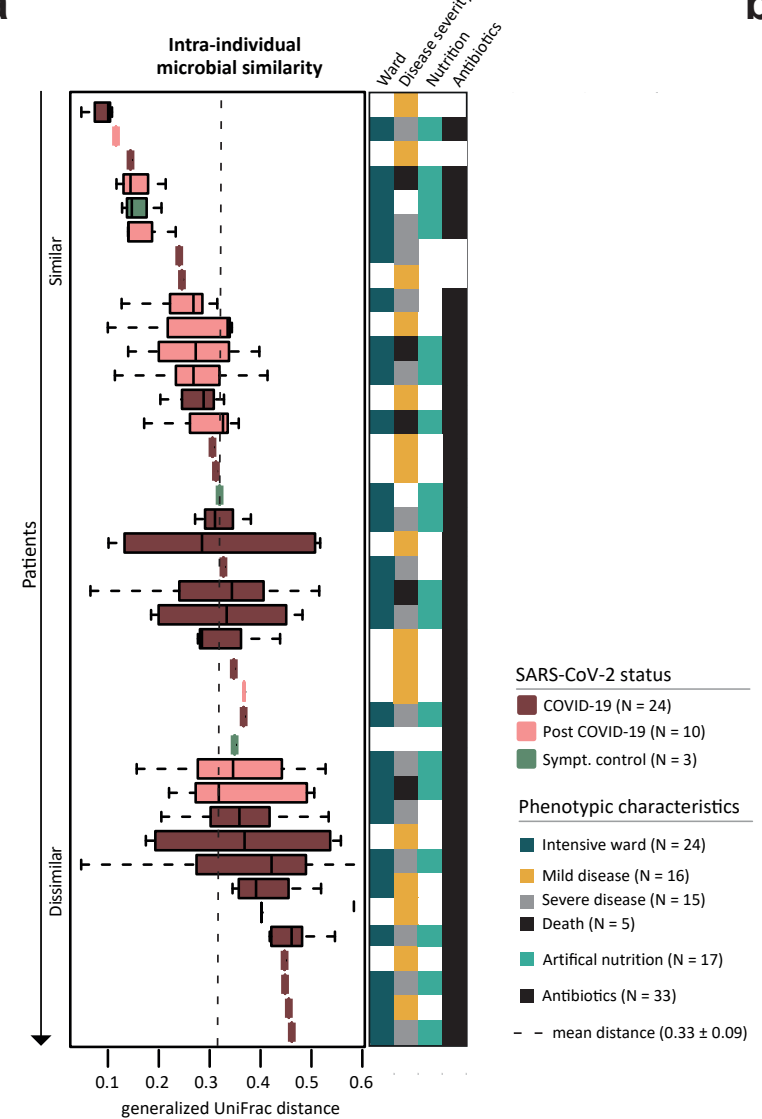

d

Severe disease progression

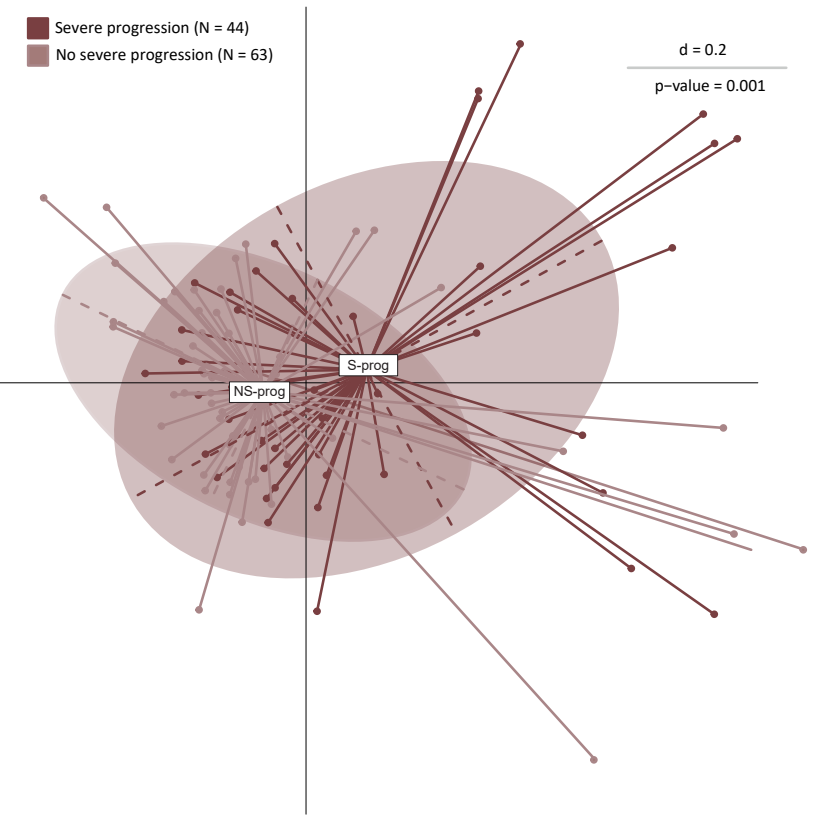

(longitudinal sampling)
Complications during in-patient stay

(longitudinal sampling)

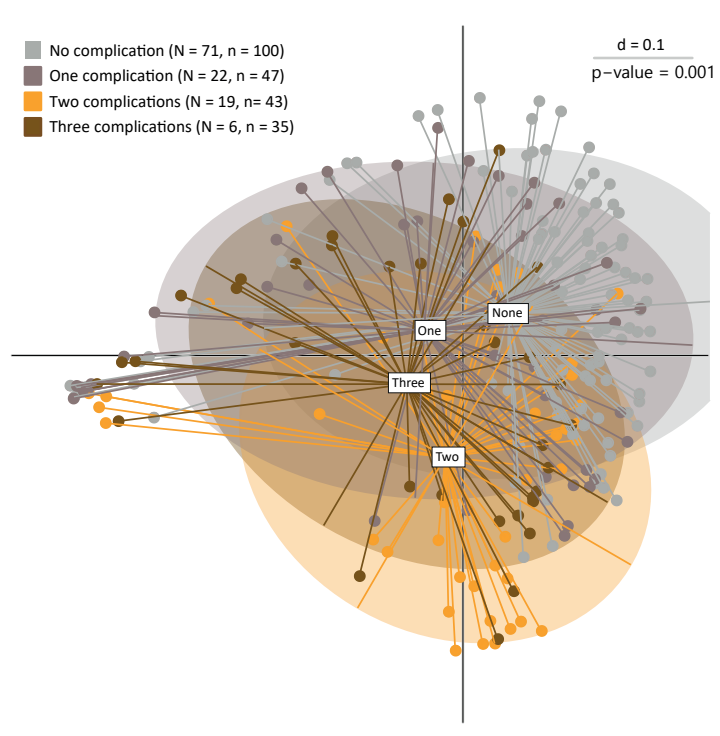

e

Random forest model trained on risk progression (longitudinal sampling)
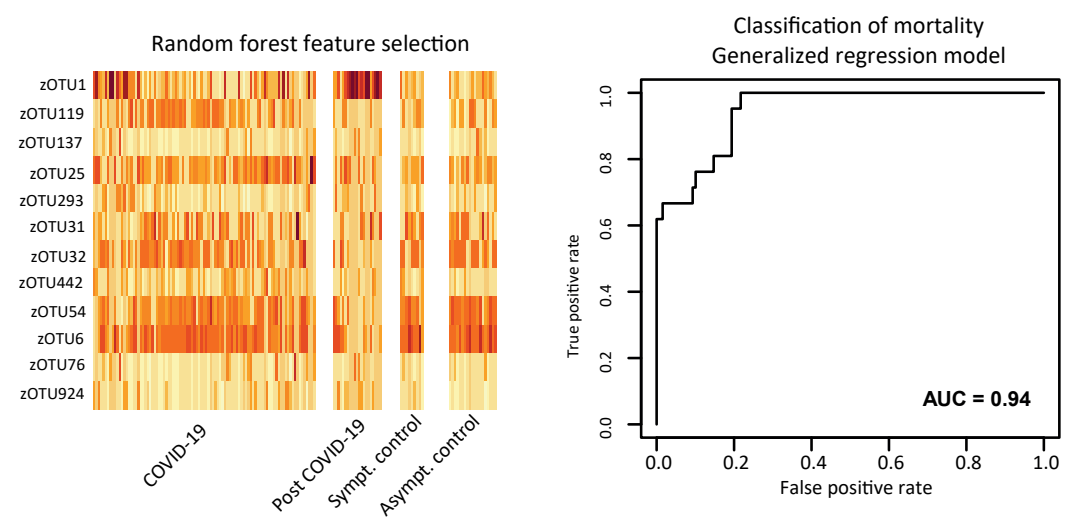
$\begin{array}{llllll}0.1 & 0.2 & 0.3 & 0.4 & 0.5 & 0.6\end{array}$

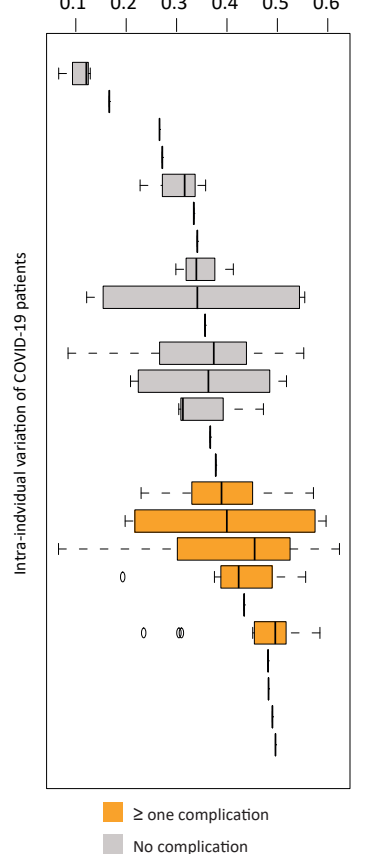

Generalized UniFrac distance 


\begin{tabular}{|c|c|c|c|c|}
\hline & COVID-19 & POST COVID-19 & SC & $A C$ \\
\hline NUMBER OF PATIENTS (N) & 108 & 22 & 20 & 26 \\
\hline STOOL SAMPLES $(N)$ & 150 & 60 & 15 & 26 \\
\hline PATIENTS WITH SERIAL STOOL SAMPLES (N) & 25 & 11 & 3 & 0 \\
\hline SALIVA SAMPLES $(N)$ & 117 & 24 & 19 & 0 \\
\hline PATIENTS WITH SERIAL SALIVA SAMPLES (N) & 15 & 5 & 2 & 0 \\
\hline GENDER (FEMALES:MALES) & 49:59 & $4: 18$ & $5: 15$ & $11: 15$ \\
\hline$A G E(Y E A R S, M E A N, S D)$ & $62(15)$ & $65(13)$ & $64(17)$ & $63(12)$ \\
\hline \multicolumn{5}{|l|}{ COMORBIDITIES (N, \%) } \\
\hline HYPERTENSION & $43(39.8)$ & $14(63.6)$ & $9(45)$ & $4(15.4)$ \\
\hline DIABETES MELLITUS II & $19(17.6)$ & $5(22.7)$ & $3(15)$ & $3(11.5)$ \\
\hline CORONARY HEART DISEASE & $16(14.8)$ & $3(13.6)$ & $8(40)$ & $1(3.8)$ \\
\hline CHRONIC KIDNEY DISEASE & $9(8.3)$ & $7(31.8)$ & $3(15)$ & $1(3.8)$ \\
\hline CANCER & $9(8.3)$ & $3(13.6)$ & $5(25)$ & $0(0)$ \\
\hline CHRONIC OBSTRUCTIVE LUNG DISEASE & $5(4.6)$ & $1(4.5)$ & $1(5)$ & $0(0)$ \\
\hline CHRONIC HEART FAILURE & $5(4.6)$ & $0(0)$ & $4(20)$ & $0(0)$ \\
\hline DIVERTICULAR DISEASE & $4(3.7)$ & $1(4.5)$ & $0(0)$ & $12(46)$ \\
\hline S.P. INTESTINAL RESECTION & $4(3.7)$ & $0(0)$ & $0(0)$ & $1(3.8)$ \\
\hline RHEUMATIC DISEASE & $4(3.7)$ & $2(9)$ & $1(5)$ & $1(3.8)$ \\
\hline INFLAMMATORY BOWEL DISEASE & $3(2.8)$ & $0(0)$ & $0(0)$ & $0(0)$ \\
\hline GASTRITIS & $3(2.8)$ & $1(4.5)$ & $0(0)$ & $4(15.4)$ \\
\hline REFLUX DISEASE & $2(1.9)$ & $1(4.5)$ & $0(0)$ & $1(3.8)$ \\
\hline \multicolumn{5}{|l|}{ SYMPTOMS AT ADMISSION (N, \%) } \\
\hline COUGH & $69(63.9)$ & $13(5.9)$ & $7(35)$ & $0(0)$ \\
\hline FEVER & $63(58.3)$ & $15(68.2)$ & $6(30)$ & $0(0)$ \\
\hline DYSPNOEA & $52(48)$ & $9(10.9)$ & $9(45)$ & $0(0)$ \\
\hline DIARRHEA & $18(16.7)$ & $7(31.8)$ & $0(0)$ & $0(0)$ \\
\hline ANOSMIA/AGEUSIA & $17(15.7)$ & $1(4.5)$ & $0(0)$ & $0(0)$ \\
\hline NAUSEA & $17(15.7)$ & $6(27.3)$ & $1(5)$ & $0(0)$ \\
\hline \multicolumn{5}{|l|}{ COMPLICATIONS DURING HOSPITALIZATION (N, \%) } \\
\hline ACUTE RESPIRATORY DISTRESS SYNDROME & $21(19.4)$ & $13(59)$ & $2(10)$ & $0(0)$ \\
\hline ACUTE KIDNEY INJURY & $17(15.7)$ & $12(54.5)$ & $4(20)$ & $0(0)$ \\
\hline ACUTE CARDIAC EVENT & $2(1.9)$ & $1(4.5)$ & $0(0)$ & $0(0)$ \\
\hline
\end{tabular}




\begin{tabular}{|c|c|c|c|c|}
\hline ACUTE PULMONARY EMBOLISM & $4(3.7)$ & $0(0)$ & $0(0)$ & $0(0)$ \\
\hline SHOCK & $3(2.8)$ & $3(13.6)$ & $1(5)$ & $0(0)$ \\
\hline PANCREATITIS & $2(1.9)$ & $0(0)$ & $0(0)$ & $0(0)$ \\
\hline VENOUS THROMBOEMBOLISM & $3(2.8)$ & $1(4.5)$ & $1(5)$ & $0(0)$ \\
\hline STROKE & $1(0.9)$ & $0(0)$ & $1(5)$ & $0(0)$ \\
\hline SECONDARY INFECTIONS (N, \%) & $54(50)$ & $19(86.4)$ & $10(50)$ & $0(0)$ \\
\hline ANTIBIOTICS $(N, \%)$ & $54(50)$ & $19(86.4)$ & $17(85)$ & $0(0)$ \\
\hline OXYGEN SUPPORT WITHOUT VENTILATION (N, \%) & $44(40.7)$ & $3(13.6)$ & $6(30)$ & $0(0)$ \\
\hline VENTILATION SUPPORT (N, \%) & $24(22.2)$ & $14(63.6)$ & $5(25)$ & $0(0)$ \\
\hline ARTIFICIAL NUTRITION (N, \%) & $17(16)$ & $12(54.5)$ & $5(25)$ & $0(0)$ \\
\hline INTENSIVE CARE (N, \%) & $30(27.8)$ & $15(68.2)$ & $5(25)$ & $0(0)$ \\
\hline IMMUNOSUPPRESSION (N, \%) & $40(37)$ & $5(22.7)$ & $3(15)$ & $2(7.7)$ \\
\hline SPECIFIC CANCER THERAPY $(N, \%)$ & $5(4.6)$ & $2(9)$ & $2(10)$ & $0(0)$ \\
\hline \multicolumn{5}{|l|}{ SPECIFIC SARS-COV-2-TREATMENT (N, \%) } \\
\hline REMDESIVIR & $15(13.9)$ & $4(18.2)$ & $0(0)$ & $0(0)$ \\
\hline CONVALESCENT PLASMA & $5(4.6)$ & $1(4.5)$ & $0(0)$ & $0(0)$ \\
\hline INTRAVENOUS IMMUNOGLOBULINS & $1(0.9)$ & $0(0)$ & $0(0)$ & $0(0)$ \\
\hline BARICITINIB & $1(0.9)$ & $0(0)$ & $0(0)$ & $0(0)$ \\
\hline
\end{tabular}

